\title{
Students' Difficulties in Transfer of Problem Solving Across Representations
}

\author{
Dong-Hai Nguyen and N. Sanjay Rebello \\ Department of Physics, 116 Cardwell Hall, Kansas State University, Manhattan, KS 66506-2601
}

\begin{abstract}
Studies indicate that the use of multiple representations in teaching helps students become better problem solvers. We report on a study to investigate students' difficulties with multiple representations. We conducted teaching/learning interviews with 20 students in a first semester calculus-based physics course. Each student was interviewed four times during the semester, each time after they had completed an exam in class. During these interviews students were first asked to solve a problem they had seen on the exam, followed by problems that differed in context and type of representation from the exam problem. Students were provided verbal scaffolding to solve the new problems. We discuss the common difficulties that students encountered when attempting to transfer their problem solving skills across problems in different representations.
\end{abstract}

Keywords: problem solving, representation, interview, transfer, physics education research PACS: $01.40 . \mathrm{Fk}$

\section{INTRODUCTION}

Research in physics education appears to indicate that the use of multiple representations helps students better understand physics ideas and solve physics problems [1]. Therefore, the ability to express physics concepts and solve problems in multiple representations is an important skill that we want our students to develop. However, it has also been shown that students have difficulties transferring their problem solving skills across representations [2].

We conducted individual teaching/learning interviews with students in a calculus-based physics course to investigate their difficulties with problem solving tasks across representations [3]. We address the following research questions in this study:

Q1. What kinds of difficulties do students have when transferring their problem solving skills across problems in different representations?

Q2. How do the difficulties depend upon the sequence in which the problems in different representations are presented?

Q3. How do these difficulties change as students progress through the semester?

\section{LITERATURE REVIEW}

Some of the early work on using multiple representations in physics education was completed by
Van Heuvelen who developed strategies to facilitate students' problem solving across representations [4]. More recently, Meltzer found that students' performance on similar problems posed in different representations might yield significantly different results [5]. Kohl et al. compared explicit and implicit teaching of multiple representations used in physics problems. He found that students learning via implicit instruction performed better and used representations more often on simple problems, whereas students learning via explicit instruction could correctly construct representations on harder problems [6].

Here we investigate the kinds of difficulties that students might have with various representational forms and with transferring their problem solving skills across representations.

\section{METHODOLOGY}

We conducted individual teaching/learning interviews with 20 students randomly selected from a pool of 102 volunteers enrolled in a first-semester calculus-based physics course [3]. Most participants were freshmen or sophomores majoring in engineering. Each student was interviewed four times during the semester, each time after they had completed an exam in their physics class.

The topics of each interview were one-dimensional kinematics in interview 1 , work and energy without 
friction in interview 2, work and energy with friction in interview 3 and rotational energy with friction in interview 4.

In interviews 2, 3 and 4, students were asked to solve three problems. These included an original problem from their most recent exam (Fig. 1), a graphical problem in which part of the information was given as a graph (Fig. 2) and a functional problem in which part of the information was given as a function (Fig. 3).

Students were asked to think aloud as they solved the problems. Hints were provided whenever students got stuck. All interviews were video and audio recorded and the students' worksheets were collected.

We used a phenomenographic approach to analyze the data from the interviews and worksheets. Data were coded for the difficulties that students expressed while solving the problems. The codes were then collapsed into categories. The inter-rater reliability between two raters for coding was about $80 \%$ before discussion and about $99 \%$ after discussion.

\section{RESULTS AND DISCUSSION}

\section{Categories of Difficulties}

A total of 17 codes were collapsed into eight categories for the difficulties that students faced. These are labeled and described below.

PRINCIPLE: Students were not sure of the appropriate principle to use to solve the problem or wrote incorrect expressions of those principles. For instance, they included only work done by friction in 'Work-Kinetic Energy' theorem and not the work done by other forces.

QUANTITY: Students used inappropriate physical quantities to describe the situation, did not know how to calculate a quantity or used wrong units of physical quantities.

FORMULA: Students apparently did not understand the meaning of a formula or expression or did not know the formula for physical quantities such as rotational inertia of a hoop or potential energy of a spring.

VALUE: Students used inappropriate values to put in a formula. For example, they used the incline length as ' $\mathrm{h}$ ' in ' $\mathrm{mgh}$ ' when calculating potential energy.

GRAPH: Students were unable to process information from the graph provided. There were two levels of difficulties here: First, students were unable to read off values from the graph, e.g. finding the value of the $\mathrm{x}$-intercept. Second, students were unable to correctly interpret the physical meaning of the graph, such as the area under the graph is the work done.
A spring of spring constant $3.0 \mathrm{kN} / \mathrm{m}$ is compressed a distance of $1.5 \mathrm{~cm}$ and a small ball is placed in front of it. The spring is then released and the small ball, mass $0.1 \mathrm{~kg}$, is fired along the slope and launched into the air at point $A$ which is $10 \mathrm{~cm}$ above the spring. The angle $\theta$ of velocity at launch is $30^{\circ}$. Friction is negligible.

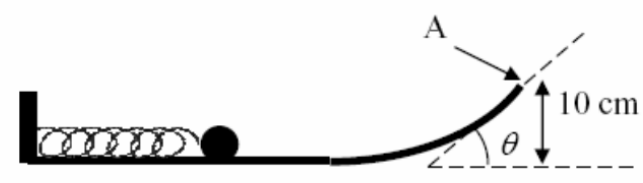

What is the speed of the ball at the launch point (point A)?

FIGURE 1. Original problem in interview 2.

A $0.1 \mathrm{~kg}$ bullet is loaded into a gun (muzzle length $0.5 \mathrm{~m}$ ) compressing a spring as shown. The gun is then tilted at an angle of $30^{\circ}$ and fired.

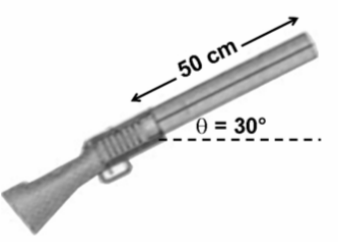

The only information you are given about the gun is that the barrel of the gun is frictionless and when the gun is held horizontal, the net force $F(N)$ exerted on a bullet by the spring as it leaves the fully compressed position varies as a function of its position $\mathrm{x}(\mathrm{m})$ in the barrel as shown in the graph below.

What is the muzzle velocity of the

bullet as it leaves

the gun, when the

gun is fired at the

$30^{\circ}$ angle as shown above?

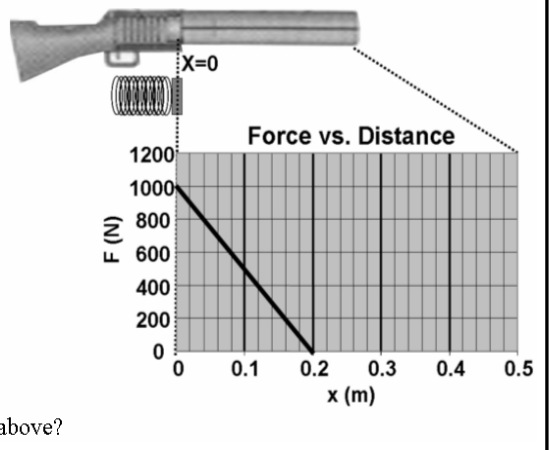

FIGURE 2. Graphical problem in interview 2.

A $0.1 \mathrm{~kg}$ bullet is loaded into a gun (muzzle length $0.5 \mathrm{~m}$ ) compressing a spring to a maximum of $0.2 \mathrm{~m}$ as shown. The gun is then tilted at an angle of $30^{\circ}$ and fired.

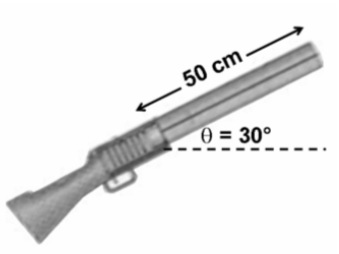

The only information you are given about the gun is that the barrel of the gun is frictionless and that the gun contains a non-linear spring such that when the held horizontal, the net force, $\mathrm{F}(\mathrm{N})$ exerted on a bullet by the spring as it leaves the fully compressed position varies as a function of the spring compression, $\mathrm{x}(\mathrm{m})$ as given by: $F=1000 x+3000 x^{2}$

What is the muzzle velocity of the bullet as it leaves the gun, when the gun is fired at the $30^{\circ}$ angle as shown above?

FIGURE 3. Functional problem in interview 2. 
MATH: Students were unable to manipulate basic mathematical processes, e.g. confusion between sine and cosine or wrong determination of angle between two vectors.

FUNCTION: Students were unable to interpret the meaning or use the function given to find the desired quantities. For instance, students substituted some values of variables into the function for force and multiplied by the total distance to find the work done rather than integrating the function.

CALCULATION: Students made simple mathematical errors in calculation such as not squaring velocity in calculating kinetic energy.

\section{Sequencing Effects}

We also investigated the effect of the sequence of the problems on students' transfer by giving half of the students the graphical problem before the functional problem, which we called the G-F sequence, and the rest of the students the functional problem before the graphical problem, which we called the F-G sequence. The average number of difficulties students had in interview 2 with the G-F and F-G sequences is shown in Fig. 5.

Overall students had more difficulties related to functions and graphs in the G-F sequence compared to the F-G sequence. This difference between the two difficulties in the two sequences was mainly due to the fact that students had significantly more difficulties with graphs in the G-F sequence compared to the F-G sequence.

It appears from these data that the functional problem, when presented before the graphical problem, assisted the students in solving the graphical problem while the graphical problem, when presented before the functional problem, does not assist the students in solving the functional problem.

\section{Trends Across Interviews}

We present the data of the average number of difficulties of each category that students encountered in interview 2 (Fig. 4) and interview 4 (Fig. 6). These students were given the graphical problem before the functional problem. In interview 2, students had significant difficulties transferring from verbal representation (original problem) to graphical representation (graphical problem). They also had difficulties with physical principles and quantities.

As seen in Fig. 6, when students went on to interview 4, the average number of difficulties with graphs decreased dramatically, which implied that students had become more comfortable working with graphs. Students were able determine the appropriate principle to use.

However, there were considerable increases in the average number of difficulties with physical quantities, formulae, values and calculations due to the increase in complexity of the problems. There were no major difficulties with the functional problems observed in these graphs. This agrees with the trend observed in Figs. 4 and 5 that the second transfer is much easier than the first one.

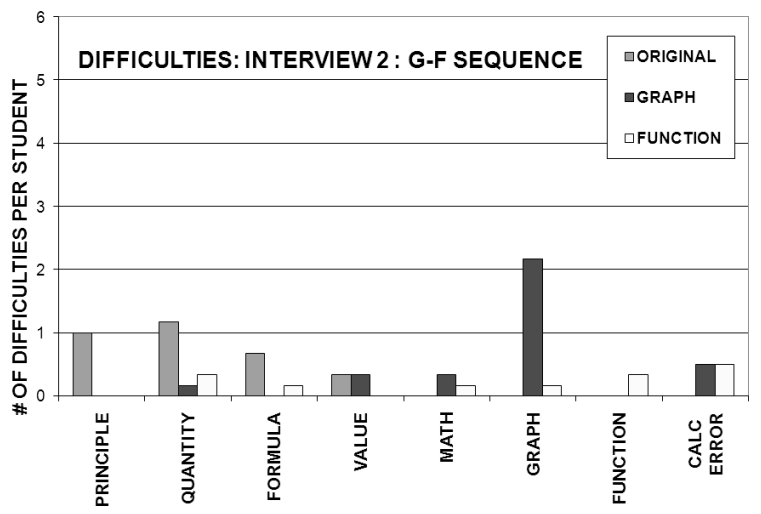

FIGURE 4. Difficulties in interview 2 when the graphical problem was asked before the functional problem.

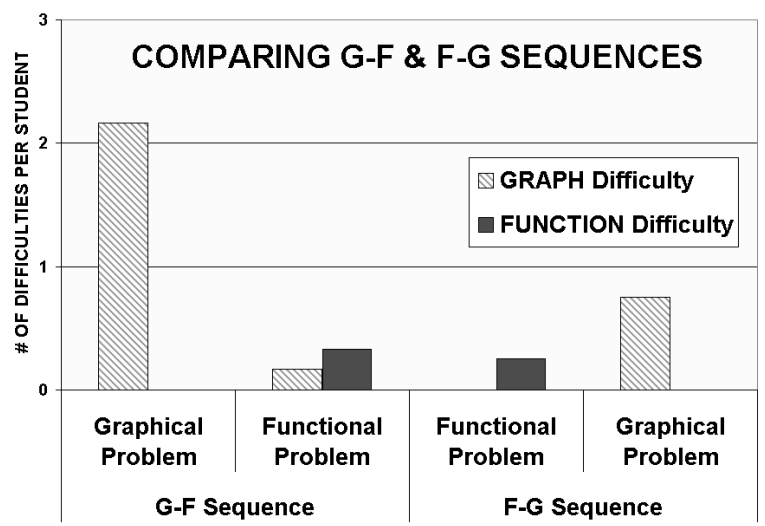

FIGURE 5. Comparing difficulties in the G-F and F-G sequences in interview 2 .

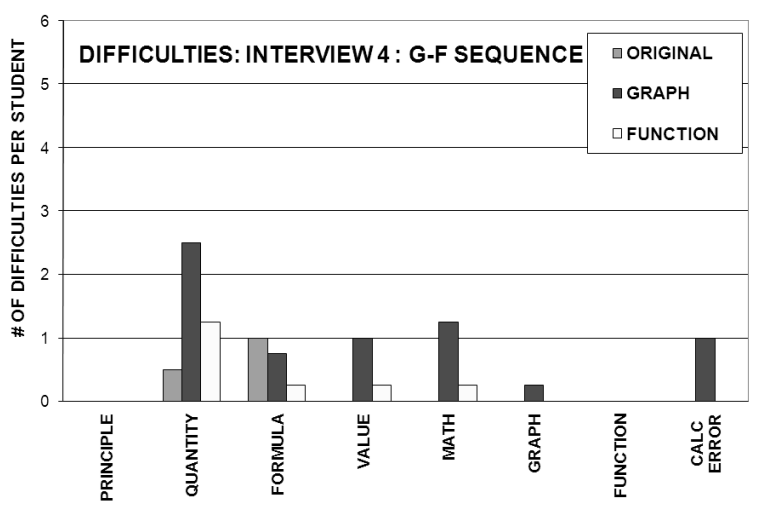

FIGURE 6. Difficulties in interview 4 when the graphical problem was asked before the functional problem 


\section{CONCLUSIONS}

We address each of the research questions below.

Q1. What kinds of difficulties do students have when transferring their problem solving skills across problems in different representations?

We found that students had significant difficulties in transferring their problem solving skills from verbal representation to graphical and functional representations. Students had difficulties extracting information from the graph, except for the coordinates of a point and the slope. When given a function, students attempted to find its value at some points rather than integrating it. Students also had considerable difficulties with interpreting physical meaning of mathematical operations such as derivative and integration although they could easily perform these operations. Many of the difficulties appeared to arise due to the tendency of students trying to mimic the previous problem when faced with a new but similar problem. This tendency seemed to be a barrier to successful problem solving in the situations explored in this study because problems in different representations required different techniques.

Q2. How do the difficulties depend on the sequence in which the problems in different representations are presented?

We also found that students' difficulties depended on the sequence in which the problems were presented. (Fig. 5) When the functional problem was presented before the graphical problem, most of the difficulties that students had were in identifying a strategy to solve a functional problem and the way to calculate physical quantities using the function given. Students appeared to face fewer difficulties in the graphical problem that followed. When the graphical problem was presented before the functional problem the major difficulties that students faced were in extracting both explicit and implicit information from the graph. Even after students had identified a way to solve the problem, they still struggled to interpret the graph and apply the information to the problem.

Q3. How do these difficulties change as students progress through the semester?

As students progressed through the semester, they had fewer difficulties transferring across representations. This trend is seen by comparing Fig. 4 with Fig. 6. This could be because students were getting used to multiple representations and they were able to apply the skills they had gained from previous interviews to the later interviews. There was still an increase in difficulties in quantities, formulae, values and calculations (Fig. 6). Students' responses to interview questions indicated that this increase was not due to the representations but due to the increase in complexity of the problems. Problems involving rotational motion in interview 4 were deemed more complex than problems involving linear motion in interview 2.

\section{Implications for Instruction}

Students encountered a variety of difficulties when transferring their problem solving across representations. However, with proper hints given by the interviewer, all students were able to solve the problems correctly. This implies that proper scaffolding from instructors might help students broaden some of their problem solving skills.

The progress of students from interview 2 to interview 4 seems to indicate that they become more competent with graphical and functional representations as they progress through the teaching/learning interviews. When asked, most of the students said that they actually learned to work with graphical and functional representations in our interviews rather than in their mathematics or physics classes. We speculate that if students had worked with problems in multiple representations more regularly, they would be more competent with different representations of the problems. Problems in multiple representations given as homework can facilitate students to build their representational competence. The sequencing effect discussed in this paper should also be considered when assigning problems of different representations to students.

\section{ACKNOWLEDGMENTS}

This research is supported in part by NSF grant 0816207. Opinions expressed are those of the authors and not necessarily those of the Foundation.

\section{REFERENCES}

1. J. I. Heller and F. Reif, Cognitive Instruction 1, 177 (1984).

2. M. W. van Someren, P. Reimann, H. P. A. Boshuizen, et al., Learning with Multiple Representations. (Pergamon Press, Oxford, U.K., 1998).

3. P. V. Engelhardt, E. G. Corpuz, D. J. Ozimek et al., presented at the Physics Education Research Conference, 2003, Madison, WI, 2003 (unpublished).

4. A. Van Heuvelen and X. Zou, American Journal of Physics 69 (2), 184 (2001).

5. D. E. Meltzer, American Journal of Physics 73 (5), 463 (2005).

6. P. B. Kohl, D. Rosengrant, and N. D. Finkelstein, presented at the 2006 Physics Education Research Conference, Syracuse, NY, 2006 (unpublished). 